\title{
A realidade do serviço público no tratamento de fratura panfacial: Relato de caso
}

\author{
The reality of public service in the treatmente of panfacial fracture: Case Report \\ La realidad del servicio público en el tratamiento de la fractura panfacial: Reporte de caso
}

Recebido: 20/05/2021 | Revisado: 28/05/2021 | Aceito: 20/07/2021 | Publicado: 28/07/2021

\author{
Luciano Martins \\ ORCID: https://orcid.org/0000-0002-2939-9970 \\ Hospital Geral de Taipas "Kátia de Souza Rodrigues", Brasil \\ E-mail: facial_surgery@hotmail.com \\ Jenifer Diana Souza da Fonseca \\ ORCID: https://orcid.org/0000-0001-5274-3832 \\ Universidade Santo Amaro, Brasil \\ E-mail: jeniferdiana123@hotmail.com.br \\ Dayane Jaqueline Gross \\ ORCID: https://orcid.org/0000-0001-6642-4672 \\ Hospital Geral de Taipas "Kátia de Souza Rodrigues", Brasil \\ E-mail: dayanejgr@hotmail.com \\ Marcelo Fernandes de Castro \\ ORCID: https://orcid.org/0000-0003-1614-7296 \\ Universidade Santo Amaro, Brasil \\ E-mail: cdmarcelocastro@hotmail.com \\ Angélica Castro Pimentel \\ ORCID: https://orcid.org/0000-0002-6317-6439 \\ Universidade Santo Amaro, Brasil \\ E-mail: draangelicacp@uol.com.br \\ Wilson Roberto Sendyk \\ ORCID: https://orcid.org/0000-0002-3742-1330 \\ Universidade Santo Amaro, Brasil \\ E-mail:wsendyk@prof.unisa.br \\ Heloisa Fonseca Marão \\ ORCID: https://orcid.org/0000-0002-6128-8576 \\ Universidade Santo Amaro, Brasil \\ E-mail: heloisafonsecamarao@yahoo.com.br
}

\begin{abstract}
Resumo
Objetivo do presente estudo é apresentar um caso clínico de fratura panfacial com tratamento híbrido através da Fixação Interna Rígida e Fixação Semi Rígida da fratura complexa mandibular. Paciente do gênero feminino, 21 anos, feoderma, internada na UTI do Hospital Geral de Taipas após acidente motociclístico, foi avaliada pelo serviço de Cirurgia e Traumatologia Buco Maxilo Facial, na qual foi diagnosticada com fratura panfacial, apresentado fraturas Le Fort I, II, III, Lannelong, fratura nasal e fratura complexa mandibular: ângulo direito e parassínfise esquerda. $\mathrm{O}$ exame de tomografia computadorizada de face confirmou a presença da fratura panfacial. Foi realizado procedimento cirúrgico de traqueostomia mais instalação de barra de Erich superior e inferior com redução incruenta da fratura mandibular com bloqueio maxilo mandibular com elástico no primeiro tempo cirúrgico. Após estabilização do quadro clínico foi realizado fixação da fratura mandibular através dos acessos, submandibular e submentual com fixação interna rígida da fratura do ângulo mandibular direito e Fixação Semi Rígida da fratura de parassínfise esquerda e bloqueio maxilo mandibular com elástico. A paciente foi transferida da UTI para enfermaria onde recebeu alta hospitalar apresentando simetria facial satisfatória, oclusão dentária Classe I de Eagle, movimentos mandibulares preservados e ausência completa após 7 meses dos sinais e sintomas de parestesia e neuropraxia dos pares cranianos V e VII.
\end{abstract}

Palavras-chave: Traumas; Fraturas; Ossos faciais; Cirurgia bucal; Traumatismo facial; Le Fort; Mandíbula.

\begin{abstract}
Objective of the present study is to present a clinical case of panfacial fracture with hybrid treatment through Rigid Internal Fixation and Semi Rigid Fixation of complex mandibular fracture. Female patient, 21 years old, feoderma, admitted to the ICU of Hospital Geral de Taipas after motorcycle accident, evaluated by the Buco Maxillofacial Surgery and Traumatology service in which she was diagnosed with panfacial fracture, with Le Fort I fractures, II, III, Lannelong, nasal fracture and complex mandibular fracture: right angle and left parasymphesis. The CT scan of the face confirmed the presence of Panfacial fracture. A tracheostomy surgical procedure was performed, plus the installation of an upper and lower Erich bar with closed reduction of the mandibular fracture with maxillary mandibular block with
\end{abstract}


an elastic band in the first surgical period. After stabilization of the clinical picture, fixation of the mandibular fracture was performed through the accesses, submandibular and submental with FIR of the fracture of the right mandibular angle and Semi Rigid Fixation of the left parasymphysis fracture and mandibular maxillary block with elastic. The patient was transferred from the UTI to the ward where she was discharged from the hospital with satisfactory facial symmetry, Eagle Class I dental occlusion, preserved mandibular movements and complete absence after 7 months of signs and symptoms of paresthesia and neuropraxia of cranial nerves V and VII.

Keywords: Traumas; Fractures; Facial bones; Oral surgery; Facial trauma; Le Fort; Jaw.

\section{Resumen}

Objetivo del presente estudio es presentar un caso clínico de fractura panfacial con tratamiento híbrido mediante la Fijación Rígida Interna y Fijación Semirrígida de la fractura compleja mandibular. Caso clínico: Paciente femenina de 21 años con feoderma, ingresada en la UCI del Hospital Geral de Taipas tras accidente de motocicleta, evaluada por el servicio de Cirugía y Traumatología Maxilofacial Buco en la que fue diagnosticada de fractura panfacial, con fracturas de Le Fort I, II, III, Lannelong, fractura nasal y fractura mandibular compleja: ángulo recto y parasíntesis izquierda. La tomografía computarizada de la cara confirmó la presencia de fractura panfacial. Se realizó un procedimiento quirúrgico de traqueotomía, más instalación de la barra de Erich superior e inferior con reducción cerrada de la fractura mandibular con bloqueo maxilar mandibular con banda elástica en el primer período quirúrgico. Posterior a la estabilización del cuadro clínico, se realizó la fijación de la fractura mandibular a través de las accesiones, submandibular y submentoniana con FIR de la fractura del ángulo mandibular derecho y Fijación Semirrígida de la fractura de la parasinfisis izquierda y bloqueo maxilar mandibular con elástico. La paciente fue trasladada de la UCI a la sala donde fue dada de alta del hospital con simetría facial satisfactoria, oclusión dental Eagle Clase I, movimientos mandibulares conservados y ausencia completa a los 7 meses de signos y síntomas de parestesia y neuropraxia de pares craneales $\mathrm{V}$ y VII.

Palabras clave: Traumas; Fracturas; Huesos faciales; Cirugía oral; Trauma facial; Le Fort; Mandíbula.

\section{Introdução}

As fraturas panfaciais são caracterizadas pelo acometimento das regiões os terços superior, médio e inferior da face com o envolvimento de diversas estruturas ósseas que podem incluir o complexo zigomático-maxilar, região naso-etimoide, região etmoidal naso-orbitária, ossos frontais, mandíbula, maxila e estruturas palato-alveolares, ainda associada a outras emergências (Degala et al., 2015; Kumar, K. A et al., 2014). Frequentemente esse tipo de fratura está associada a lesões de tecido mole, deformidades faciais e má oclusão, com perda de altura ou projeção facial, largura facial aumentada e enoftalmia (Banerjee et al., 2016). Lesões crânio-cerebral e lesão da coluna cervical, ampliando a complexidade do trauma de etiologia variada (Singh et al., 2015).

Entre os fatores etiológicos da fratura incluem-se os traumas de acidentes automobilísticos de alto impacto, traumas esportivos, quedas relacionadas a acidente de trabalho e ferimento por arma de fogo. Estas causas estão entre as ocorrências mais significativas de fraturas panfaciais, caracterizando-se em uma redução desafiadora para o cirurgião e para a equipe multidisciplinar (Chaudhry et al., 2018; Yun et al., 2019; Degala et al., 2015).

O tratamento das lesões panfaciais incluem a atuação multiprofissional com a avaliação das condições que comprometam a vida, avaliação das vias aéreas, controle da consciência, controle de sangramento e o tratamento de lesões associadas tendo o manejo e a proteção das vias aéreas em extrema importância (Ali et al., 2017; Guerrissi, 2016). Encontra-se na literatura diversas abordagens para o tratamento das fraturas panfaciais, o qual pode ser um grande desafio para o cirurgião, devido à ausência de um ponto fixo para estabilização. O sucesso do tratamento envolve a compreensão dos componentes da fratura e a capacidade de relacioná-los anatomicamente aos elementos ósseos estáveis, tendo como referência a largura, altura e projeção facial (Choi et al., 2019).

Com a evolução tecnológica para o planejamento tridimensional e a possibilidade de fixação rígida com placas e parafusos, tornou-se possível a variação da sequência da redução baseado no planejamento individual que através de softwares virtuais e com o auxílio das tomografias computadorizadas possibilitando a visualização e planejamento que associados ao exame clínico, otimizam os resultados (Ali et al., 2017; Cascone et al., 2008; Degala et al., 2015;). O desafio maior está na escolha do 
tratamento quando não encontramos os materiais atuais (placas e parafusos) para a fixação, tendo que lançar mão de métodos de tratamentos alternativos. Dessa forma, o objetivo do trabalho foi relatar um caso de fratura mandibular complexa tratada de forma híbrida com FIR (fixação interna rígida) e fixação semi-rígida em uma paciente com diagnóstico de fratura panfacial, onde técnicas antigas de redução foram indicadas com resultados altamente satisfatórios.

\section{Metodologia}

Este é um estudo descritivo do tipo relato de caso (Pereira et al., 2018), em que foi feito a descrição do tratamento e conduta de uma paciente que apresentava fratura panfacial e foi admitida em um hospital público estadual na cidade de São Paulo.

Um Termo de Consentimento Livre e Esclarecido (TCLE) abordando os aspectos éticos, esclarecimentos sobre riscos, benefícios e prognósticos foi fornecido ao paciente, e o mesmo autorizou a realização do procedimento, o uso de informações do prontuário e uso de imagens com finalidade acadêmica, mediante assinatura do documento.

\section{Relato do Caso}

Paciente do gênero feminino, feoderma, 21 anos, vítima de acidente motociclístico, foi encaminhada para o serviço de atendimento Hospitalar do Hospital Geral de Taipas e ao exame clínico e radiográfico foi diagnosticado trauma crânio encefálico (TCE) e fratura panfacial. Em seguida, a paciente foi encaminhada para Unidade de Terapia Intensiva (UTI) e solicitado avaliação ao serviço de Cirurgia e Traumatologia Buco-Maxilo-Facial do hospital (Figura 1).

Figura 1: Admissão Hospitalar.

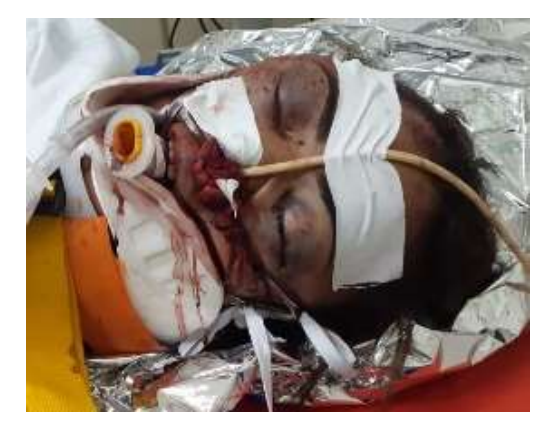

Fonte: Autores.

Foi observado análise de coleção hemorrágica heterogênea na convexidade cerebral à esquerda, com até $0,7 \mathrm{~cm}$ de espessura e múltiplas fraturas do esqueleto facial, fratura Le Fort I, II, III, fratura Lannelong, fratura nasal e fratura complexa mandíbular: ângulo direito e parassínfise esquerda (Figuras 2A, 2B e Figura 3A, 3B). 
Figura 2: Tomagrafia computadorizada (corte axial): (A) fratura em região zigomática; (B) fratura em região de ângulo mandibular e parassínfise.
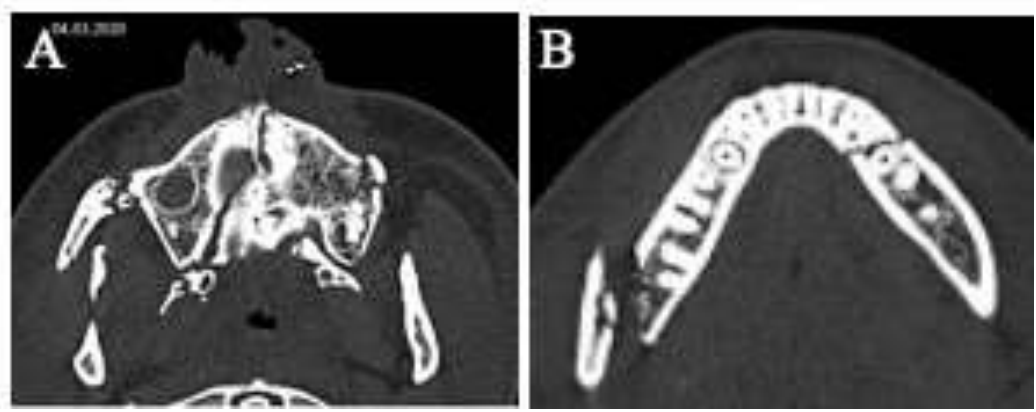

Fonte: Autores.

Figura 3: Tomagrafia computadorizada (corte coronal): (A) fratura em região zigomática; (B) fratura em região mandibular.
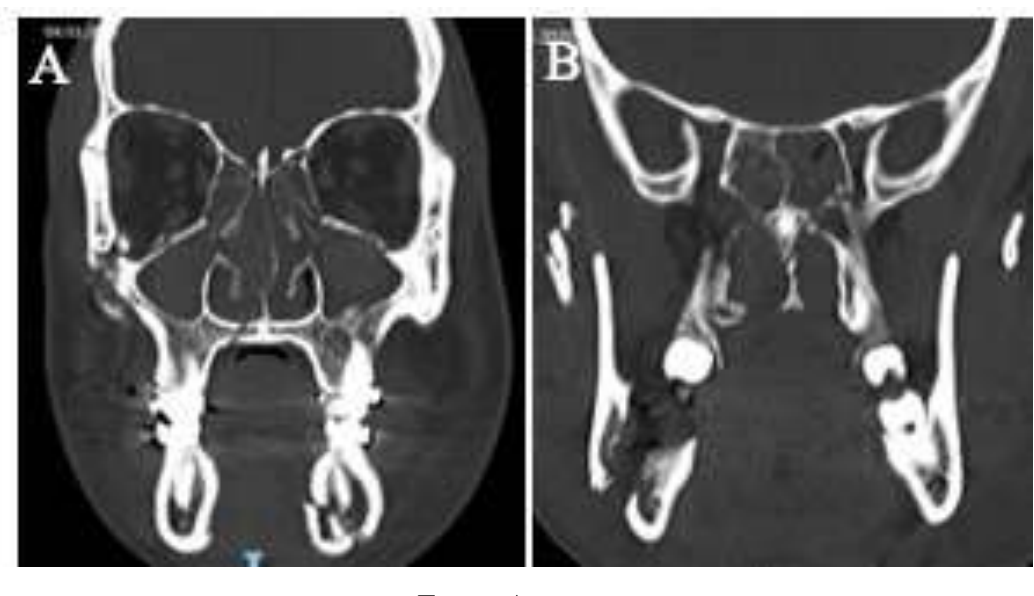

Fonte: Autores.

Após estabilização do quadro clínico da paciente na UTI, foi encaminhada para o procedimento de traqueostomia (TQT), redução incruenta e estabilização da fratura mandíbular (ângulo direito e parassínfise esquerda) e fratura sagital de maxila (Lannelong) através da instalação de barras de Erich superior e inferior, e bloqueio maxilo mandibular em classe I de Angle com elástico (Figura 4 e Figura 5A, 5B).

Figura 4: Bloquei maxilomandibular.

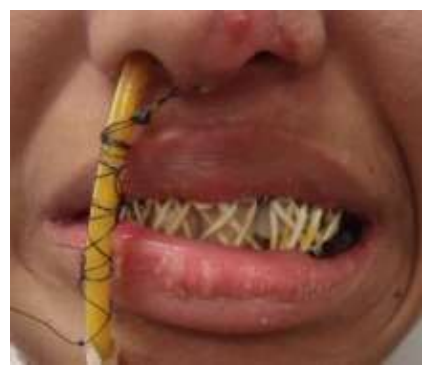

Fonte: Autores. 
Figura 5: (A) Reconstrução 3D; (B) Radiografia PA de face evidenciando a instalação da Barra de Erich e bloqueio maxilomandibular.

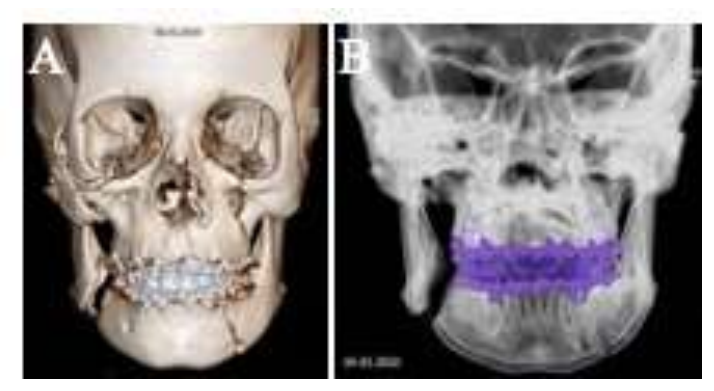

Fonte: Autores.

No pós-operatório de 15 dias, a paciente foi encaminhada para o centro cirúrgico para realização da FIR da fratura de ângulo direito mandibular com duas placas do sistema $2.0 \mathrm{~mm}$, através do acesso cirúrgico de Risdon e fixação interna semirrígida com fio de aço $\mathrm{n}^{\circ} 01$ na região da fratura de parassínfise através do acesso cirúrgico submentual e fixação em " $\mathrm{X}$ " (Figura 6).

Figura 6: Acesso de risdon e instalação de 2 placas $2.0 \mathrm{~mm}$.

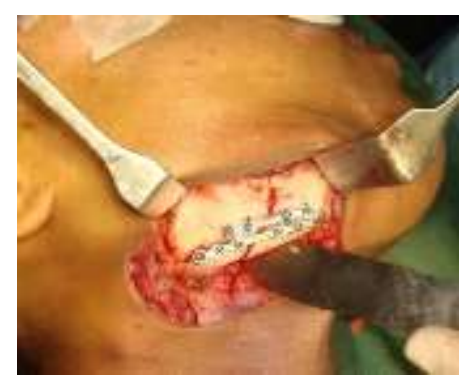

Fonte: Autores.

Foi realizado também redução incruenta da fratura nasal com tamponamento nasal anterior com gaze e neomicina; e gaze gessada no dorso nasal. As fraturas Le Fort I, II e III foram tratadas de forma conservadoras, pois não apresentavam deslocamento significativo e deformidade crânio facial que justificasse intervenção cirúrgica.

A paciente foi acompanhada mensalmente e após 45 dias do procedimento da realização do bloqueio maxilomandibular (BMM) o mesmo foi removido, onde obteve-se abertura oral de $35 \mathrm{~mm}$, movimentos mandibulares preservados (lateralidade, protrusão, retrusão) e oclusão dentária em classe I de Eagle. A paciente não relatou queixas álgicas locais, presença de infecção, dacriocistite, oftlalmoplegia, porém apresentava parestesia do VII par craniano hemifacial à direita, com comprometimento muscular do lábio inferior nos movimentos de contração.

Foi realizado controle de imagens no pós-operatório imediato, assim como em 30, 60 e 90 dias no pós-operatório através das técnicas radiográficas de imagens: radiografia de Waters, Waters reversa, Hirtz, PA de mandíbula boca fechada e boca aberta, lateral oblíqua de mandíbula direita e esquerda, radiografia panorâmica e tomografia computadorizada de face sem contraste. Todos os exames apresentaram redução satisfatória (Figura 7A E 7B). 
Figura 7: (A) e (B) Radiografia pós operatória evidenciando a correta redução e alinhamento ósseo.

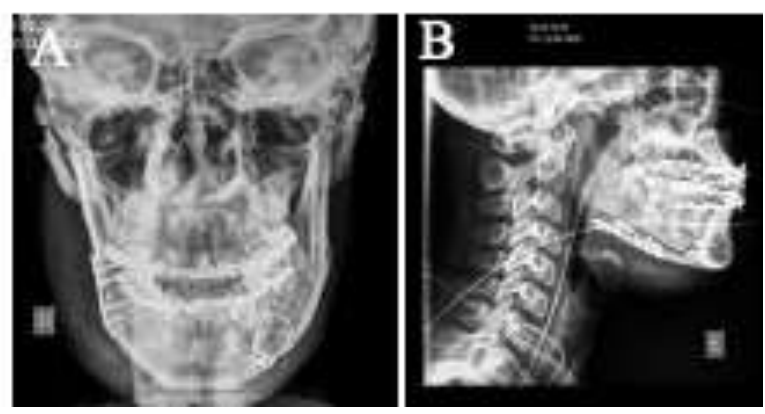

Fonte: Autores.

No pós-operatório de sete meses a paciente não apresentava sintomas de neuropraxia e parestesia. A oclusão classe I de Eagle foi mantida, paciente apresentava abertura oral de $45 \mathrm{~mm}$ e movimentos mandibulares preservados recebendo alta definitiva (Figura 8A e 8B).

Figura 8: (A) Radiografia panorâmica evidenciando correta redução das fraturas; (B) Pós-operatório mostrando adequado restabelecimento oclusal.
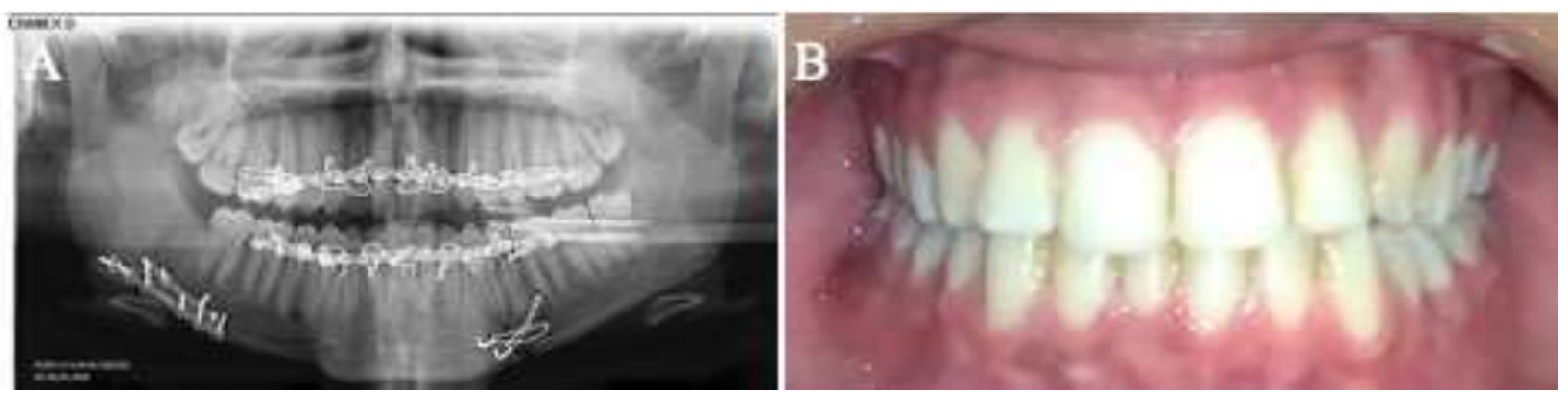

Fonte: Autores.

\section{Discussão}

O manejo de fraturas panfaciais é complexo e cada paciente requer um plano de tratamento individualizado (Koraitim, M., 2020; Sharma et al., 2015). Existem pelo menos 4 abordagens de tratamento diferentes, sendo "de fora para dentro, "dentro para fora", "superior para inferior" e "inferior para superior". Muitos cirurgiões fazem combinação destas (Ali et al., 2017; Choi et al., 2019), ou até mesmo lançam mão de abordagens próprias. Os princípios para a redução foram popularizados na década de 1980 com a proposta de ampla exposição e visualização direta das zonas da fratura. Esses princípios passaram a ser aplicados às fraturas panfaciais influenciando sua ordem de redução, com estratégias de manejo conservador diante de deslocamentos mínimo de ossos ou cirurgia de campo aberto em quadros mais complexos, o que varia entre os casos (Clauser, L et al., 2003; Chaydhry, O et al., 2015; Ramakrishnan, k., 2020).

Dos diversos tipos de tratamentos, os conservadores como o BMM, quando bem indicados, podem oferecer aceitáveis resultados (Cascone et al., 2008). O BMM é efetivo e proporciona cicatrização dos fragmentos ósseos através da redução, imobilização e estabilização, fazendo com que o meio se torne favorável para as células odontogênicas provenientes da medula óssea e periósteo atuarem na regeneração. Tal forma de tratamento, é útil para aproximar e imobilizar segmentos de fraturas cominutivas. O BMM, como através do uso da Barra de Erich citado em nosso relato, apresenta grande número de ganchos para tração elástica, fazendo com que haja uma equilibrada distribuição da tensão elástica sobre o arco. Apesar de efetivo, entre suas 
desvantagens encontra-se trauma ao periodonto, higiene oral prejudicada, risco de contaminação através da perfuração de luva, além de tempo de trabalho extenso e possível extrusão com consequente perda de vitalidade do elemento dental (Hashemi et al., 2011).

Na literatura, encontra-se o uso métodos de fixação não rígidas ou semi-rígidas há mais de 200 anos (Ellis., 1991). As fixações semi-rígidas eram realizadas por meio de fios de aço, porém era verificado que não promovia suficiente estabilidade dos fragmentos ósseos e era quase sempre complementada com o uso de bloqueio maxilo-mandibular, o que proporcionava a verdadeira estabilização interfragmentária. Os fios de aço apenas serviam para realinhar os segmentos ósseos (Ellis., 1993; Leach et al., 1995), demonstrando dessa forma, ser eficiente na restabilização óssea, como foi indicado neste caso clínico, na região parassínfise esquerda.

Com o advento das técnicas de FIR, a intervenção das fraturas mudou significativamente. A evolução dos sistemas de fixação levou ao desenvolvimento de placas de compressão, baseado no princípio de que a compressão entre os fragmentos ósseos aumenta a área de contato, promovendo cicatrização por primeira intenção (Ellis., 1993) Além disso, passou a permitir a estabilização dos segmentos da fratura, eliminando ou reduzindo a aplicação do BMM. Suas principais vantagens referem-se a maior estabilidade e precisão, função em pós-operatório imediato e reabilitação mais rápida. Essa modalidade de tratamento é indicada em situações de fraturas complexas e desfavoráveis. É um método que permite estabilizar os segmentos osteotomizados por meio de parafusos ou placas metálicas. Além de serem considerados métodos de fixação mais estáveis, possuem propriedades biomecânicas superiores às contenções com fios de aço, os quais eram anteriormente utilizados, já que permite a estabilização dos segmentos com manutenção da função mandibular durante o reparo ósseo (Mendes et al., 2011), sendo o material de fixação de escolha, sempre que disponível. Apesar de ter efeito benéfico relativamente rápido ao paciente traumatizado, o uso de FIR possui maior risco de infecções, além de ser um tratamento mais caro pelas suas placas e parafusos, o mesmo exige hospitalização e procedimento anestésico geral (Hashemi et al, 2011; Leach et al., 1995;).

Os desafios do tratamento requerem a atuação de cirurgiões experientes que pode incluir na equipe o cirurgião plástico, oftalmologista, neurologista e cirurgiões de trauma, para determinar as técnicas anestésicas, abordagens de intubação e realizar o manejo na redução, com objetivo de devolver a arquitetura facial, evitando novas intervenções ou a procedimentos que possam levar a deformidades persistentes (Chaudhry, O., 2018; Follmar, K.E et al., 2007; Kim, J at el., 2016; Ramakrishnan, k et al., 2020; Sharma, S et al., 2015). O desafio ainda é maior quando não se obtém placas e parafusos para a fixação e abordagem adequada do paciente. Diante da evolução dos materiais utilizados, espera-se que tenhamos sempre disponível os materiais necessários que ofereçam as vantagens aos pacientes. Porém, verificamos que independente da evolução dos materiais, métodos anteriores de fixação apresentam qualidades que diante da ineficiência de disponibilidade de recursos ideias, são vantajosos para oferecer um tratamento adequado ao paciente.

\section{Conclusão}

Mesmo após inúmeros avanços nas técnicas de fixações e os benefícios das modalidades de tratamento mais atuais, as antigas técnicas ainda devem ser levadas em consideração, pois no sistema de saúde pública brasileiro, ocorre uma realidade frequente que é a impossibilidade de acesso à materiais de primeira escolha para um tratamento mais adequado conforme o caso de cada paciente. Dessa forma, diante da necessidade de reabilitar o paciente e diminuir o risco de agravos, os tratamentos das

Fraturas com técnicas de fixação semi-rígidas e bloqueio maxilo mandibular evidenciam a necessidade do conhecimento dessas técnicas pelos Cirurgiões e Traumatologistas Bucomaxilofaciais.

Sugerimos para trabalhos futuros a importância da comparação entre técnica semi-rígida versus fixação interna rígida em fraturas mandíbulares 


\section{Agradecimentos}

Agradeço o empenho e a colaboração do Dr. Jaci Francisco Martins, médico, cirurgião geral membro adjunto do Colégio Brasileiro de Cirurgiões, especialista em endoscopia digestiva e membro titular pela Sociedade Brasileira de Endoscopia Digestiva (SOBED) que não mediu esforços para o sucesso do caso reportado.

\section{Referências}

Ali, K., Lettieri, S.C. (2017). Management of Panfacial Fracture. Semin Plast Surg, 31(2),108-117.

Banerjee, P. K., Jain, A., Behera, B. (2016). Submandibular intubation as an alternative for intra-operative airway management in maxillofacial fractures - our institutional experience. Indian J Anaesth, 60(1), 573-577.

Cascone, P., Spallaccia, F., Graziana, M. A.F., Rivaroli, A., Saltalrel, A., Iannetti, G. (2008). Rigid versus semirigid fixation for condylar fracture: experience with the external fixation system. J Oral Maxillofac Surg, 66(2), 265-271.

Chaydhry, O., Isakson, M., Franklin A., Maqusi, S., El Amm, C. (2015). Facial Fractures: Pearls and Saikrishna Degala, S. Shyam Sundar, K. S. Mamata. A Comparative Prospective Study of Two Different Treatment Sequences i.e. Bottom Up-Inside Out and Topdown-Outside in, in the Treatment of Panfacial Fractures. J Maxillofac Oral Surg, 14(4), 986-994.

Choi, J. W., Kim, M. J. (2019). Treatment of Panfacial Fractures and Three-Dimensional Outcome Analysis: The Occlusion First Approach. J Craniofac Surg, 30(4), 1255-1258.

Clauser, L., Galiè, M., Mandrioli, S., Sarti, E. (2003). Severe panfacial fracture with facial explosion: integrated and multistaged reconstructive procedures. $J$ Craniofac Surg, 14(6):893-988.

Degala, S., Sundar, S.S., Mamata, K.S. (2015). A Comparative Prospective Study of Two Different Treatment Sequences i.e. Bottom Up-Inside Out and Topdown-Outside in, in the Treatment of Panfacial Fractures. J Maxillofac Oral Surg, 14(4), 986-994.

Ellis, E., Dechow, P.C., Carlson, D.S. (1988). A comparison of stimulated bite force after mandibular advancement using rigid and nonrigid fixation. J. oral maxillofac. Surg, Philadelphia. 46(1), 26-32.

Ellis, E. (1991). The internal fixation of fractures: Historical perspectives. In: TUCKER, M.R., et al. Rigid Fixation for Maxillofacial Surgery. Philadelphia: 1 B. Lippincot, Parte I, cap.I, 3-29.

Filho, J., França, S., Araújo, L., Pereira, J., Belchior, I., \& Sampieri, M. (2018). Intervenção cirúrgica de um canino inclu so em sínfise mandibular: relato de caso. Revista Da Faculdade De Odontologia - UPF, 23(3), 329-332.

Follmar, K. E., Debruijn, M., Baccarani, A., Bruno, A. D., Mukundan, S., Erdmann, D., Marcus, J.R. (2007). Concomitant injuries in patients with panfacial fractures. J Trauma, 63(4), 831-835.

Guerrissi, J. O. (2016). Treatment Options in Maxillofacial Fractures. J Craniofac Surg, 27(5), 445-447.

Hashemi, H. M., Parhiz, A. (2011). Complications using intermaxillary fixation screws. J Oral Maxillofac 577 Revista da Universidade Vale do Rio Verde, Três Corações.10(1), 570-578.

Jang, S. B., Choi, S. Y., Kwon, T. G., Kim, J. W. (2020). Concomitant injuries and complications according to categories of pan-facial fracture: a retrospective study. J Craniomaxillofac Surg, 48(4),427-434.

Kumar, K.A., Kumar, B. P., Mohan, A. P., Masram, A. K., Tyro, D., Gandla, D. (2015). Assessment of the Efficacy of Submental Intubation in the Management of Midfacial and Panfacial Trauma Patients. J Maxillofac Oral Surg. 14(3), 674-81.

Kim, J., Choi, J. H., Chung, Y. K., Kim, S. W. (2016). Panfacial Bone Fracture and Medial to Lateral Approach. Arch Craniofac Surg, 17(4). 181-185.

Koraitim, M. (2020). Panfacial Fractures Management. J Craniofac Surg, 31(4), 329-331.

Leach, J., Truelson, J. (1995). Traditional Methods vs Rigid Internal Fixation of Mandible Fractures. Arch Otolaryngol Head Neck Surg, 121(7), 750-753.

Mansher, S., Joseph, A., Ricci, E. J. C. (2015). Use of Intraoperative Computed Tomography for Revisional Procedures in Patients with Complex Maxillofacial Trauma. Plast Reconstr Surg Glob Open, (3), 463.

Mendes, R. B., Dultra, J. D. A., Dultra, F. K. A., Maciel, A. D. S., Toledo, Í. C., Azevedo, R. A. (2011). Evaluation of conservative treatment of mandibular fracture. Int J Oral Maxillofac Surg, 40(10),1113.

Ramakrishnan, k., Palanivel, I., Narayanan, V., Chandran, S., (2020). Narayanan, J. Sequencing of Fixation in Panfacial Fracture: A Systematic Review. J Maxillofac Oral Surg, 20(2), 180-188.

Singaram, M., Ganesan, I., Kannan, R., Kumar, R. (2016). Avaliação da segurança e utilidade da intubação submentoniana na cirurgia de trauma panfacial. $J$ Korean Assoc Oral Maxillofac Surg, 42(2), 99-104.

Sharma, S, Dhanasekaran, V. (2015). Surgical Approaches and Management of Panfacial Trauma: A Case Report. J Clin Diagn Res, 9(8), $13-14$.

Yun, S., Na, Y. (2018). Panfacial bone fracture: cephalic to caudal. Arch Craniofac Surg, 19(1), 1-2. 